\title{
Development of Medium Duration, High Oil Content and Yield Potential Groundnut Variety Konkan Bhuratna for Konkan Region of Maharashtra State, India
}

\author{
B.D. Waghmode ${ }^{1 *}$, V.C. Navhale ${ }^{1}$, A.S. Kamble ${ }^{1}$, N.G. Sonone ${ }^{1}$ and B.L. Thaware ${ }^{2}$ \\ ${ }^{1}$ Agricultural Research Station, Shirgaon, Ratnagiri - 415629 (MS), India \\ ${ }^{2}$ Department of Agricultural Botany, Collage of Agriculture, Dapoli-415 712, Maharashtra, India \\ *Corresponding author
}

A B S T R A C T

Keywords

Konkan Bhuratna, High yield, High oil percent, Resistance to diseases and pest.

Article Info

Accepted:

10 October 2017

Available Online:

10 December 2017
The groundnut variety, Konkan Bhurtana (RTNG 29) was evolved from the cross between PBS 24030 and GPBD 4 using former parent through hybridization followed by pedigree method of selection. Konkan Bhuratna variety is medium duration (115-120 days), Virginia Bunch type groundnut culture having 2.5 to 3.0 t/ha average pod yield, with $50.01 \%$ oil content, $23.44 \%$ protein content and $74 \%$ shelling percentage, it is medium bold kernel type variety, suitable for both kharif and rabi season. It is resistant to early and late leaf spot, rust, PBND, alterneria leaf blight diseases and also resistant to thrips, jassids, leaf miner, defoliator insect and pests. Therefore the groundnut variety Konkan Bhuratna recommended for release for commercial cultivation in upland area during Kharif and irrigated area during Rabi season in Konkan region of the Maharashtra state during the year 2017.

\section{Introduction}

Groundnut is considered to be the one of the most important oilseed crops in the world. It is cultivated in more than 100 countries on $26.54 \mathrm{~m}$ hectare area with an annual production of $43.91 \mathrm{~m}$ tonnes and productivity of $1655 \mathrm{~kg} / \mathrm{ha}$ (FAOSTAT, 2015). In India, groundnut is grown on $4.77 \mathrm{~m}$ hectare area with the production of $7.40 \mathrm{~m}$ tonnes (FAOSTAT, 2015). The productivity of groundnut in India is low $(1552 \mathrm{~kg} / \mathrm{ha})$ compared to Israel $(7389 \mathrm{~kg} / \mathrm{ha})$, USA (4397 $\mathrm{kg} / \mathrm{ha}$ ), China (3492 kg/ha) and Argentina (2848 kg/ha) (FAOSTAT, 2015). Groundnut kernels are regarded as healthy foods as their nutrient profile is balanced (Arya et al., 2016). The kernels contain $48-50 \%$ oil, 10 $20 \%$ carbohydrates, and $25-28 \%$ easily digestible protein, and provide $564 \mathrm{kcal}$ of energy for every $100 \mathrm{~g}$ of kernels (Arya et al., 2016)

In Maharashtra state it is cultivated on area of 1.96 lakh hectares with productivity of 1163 $\mathrm{kg} / \mathrm{ha}$ during kharif season and 0.71 lakh ha area with $1366 \mathrm{~kg} / \mathrm{ha}$ productivity during rabi season 2014-15. In Konkan region groundnut is grown on about 20,000 ha area with 1.8 t/ha productivity. The groundnut crop is new in 
this region. However, the area under konkan is increased enormously. The upland early duration rice varieties are being replacing with groundnut cultivation due to poor yield in rice. The groundnut is increasing fastly during rabi season in commanded areas of different dams and rivers in konkan region. The soils are porous, rich in potash, optimum temperature and humidity favors higher pod yields in groundnut which is double as compared to upghat region of rest of Maharashtra.

Therefore, it was essential to devolved, high yielding, medium duration, high shelling percentage with high oil content and having fresh seed dormancy more than 21 days, resistant to major diseases and pests variety for cultivation in groundnut growing tracts of Konkan region of Maharashtra state. With considering the above points the Konkan Bhurtana is developed.

\section{Materials and Methods}

It is cross between PBS 24030 and GPBD 4. The selections were made for high oil, fresh seed dormancy, resistance to major disease, pest and high yielding progenies from the segregating generations of above cross. Among the several selections in segregating populations of above cross, a promising pure line during 2010 to 2012 RTNG-29 was selected and further tested in various trials viz., station trials, university trials, state coordinated trials and national co-ordinated trials at various locations in region at state during 2012 to 2016. This culture also tested in agronomical at Agricultural Research Station, Shirgaon, Ratnagiri, MS during Rabi 2015-16 and Kharif 2016. Total 21 and 20 adoptive trials were conducted during Rabi 2015-16 and Kharif season 2016 respectively, in five districts of Konkan region of Maharashtra state. The culture was screened for resistance to various insect pests and diseases at endemic sites. The oil quantity and quality parameters were analyzed at Gujarat Laboratory, Gujarat. The pod yield data of various trials were statistically analyzed according to Panse and Sukhatme (1967). Based on pod yield data of various trials, superior kernel yield, resistance for disease and insect pest reactions and stable yield performance at various test locations, Konkan Bhurtan groundnut variety is recommended to release in the state of Maharashtra for commercial cultivation during the year 2017 .

\section{Results and Discussion}

The characteristic features of Konkan Bhuratna (RTNG-29) recorded as per DUS guideline at the research stations were presented in Table 1. The groundnut variety RTNG-29 is erect in growth habit, leaflet colour dark green, leaf size small $(2.6 \mathrm{~cm})$, medium duration (115-120 days), high shelling \% (>75\%), Kernel colour tan \& Shape cylindrical, Medium bold (100 Kernel wt. $48 \mathrm{~g}$ ), fresh seed dormancy (26 days) and high oil content $(50.01 \%)$.

The yield performance of Konkan Bhuratna groundnut variety in various trials conducted during 2012 to 2016 is presented in Table 2. Konkan Bhuratna (RTNG 29) groundnut variety recorded $35.81 \%$ higher pod yield (4852 kg/ha) over check TKG-Bold (3577 $\mathrm{kg} / \mathrm{ha}$ ) in station trial (Rabi) conducted during Rabi 2012-13 to 2014-15, similarly, during kharif 2013 to 2015 in station it has recorded $4358 \mathrm{~kg} / \mathrm{ha}$ pod yield which was $58.30 \%$ higher than the check TKG-Bold (Anonymous, 2017).

In university multilocation trials conducted on university farm at 7 different location Konkan Bhuratna has been recorded 19.81\% (3731 $\mathrm{kg} / \mathrm{ha}$ ) higher pod yield over the check TKGbold $(3114 \mathrm{~kg} / \mathrm{ha})$ in two season both in Kharif and Rabi. 
Table.1 Characteristic of groundnut culture, RTNG-29 as per DUS guidelines

\begin{tabular}{|c|c|c|c|c|c|}
\hline \begin{tabular}{|l|} 
S. No \\
\end{tabular} & Characteristics & RTNG-29 & S. No & Characteristics & RTNG-29 \\
\hline $\begin{array}{ll}1 . \\
(*)\end{array}$ & Plant: Growth habit & \multirow[t]{4}{*}{ Erect } & \multirow[t]{4}{*}{ 11. (*) } & $\begin{array}{l}\text { Pod: Number of kernels } \\
\text { (on } 100 \text { pod basis) }\end{array}$ & \multirow[t]{4}{*}{$\begin{array}{c}>60 \% \\
2 \text { seeded }\end{array}$} \\
\hline \multirow[t]{3}{*}{$(+)$} & \multirow{3}{*}{\begin{tabular}{r|} 
Erect \\
Semi- spreading \\
Spreading
\end{tabular}} & & & $>60 \% 2$ seeded & \\
\hline & & & & & \\
\hline & & & & $>60 \% 4$ seeded & \\
\hline \multirow[t]{4}{*}{$\begin{array}{ll}2 . \\
(*)\end{array}$} & $\begin{array}{l}\text { Leaflet: Size(fully developed } \\
\text { basal leaflet) }\end{array}$ & \multirow[t]{4}{*}{$\begin{array}{c}\text { Small } \\
(2.6 \mathrm{~cm})\end{array}$} & \multirow{4}{*}{$\begin{array}{l}12 . \\
(*) \\
(+)\end{array}$} & Pod: Presence of beak & \multirow[t]{4}{*}{ Present } \\
\hline & Small $(<4.0 \mathrm{~cm})$ & & & Absent & \\
\hline & Medium $(<4.0-6.0 \mathrm{~cm})$ & & & Present & \\
\hline & Large $(>6.0 \mathrm{~cm})$ & & & & \\
\hline \multirow[t]{4}{*}{3.} & Leaflet: Colour & \multirow{4}{*}{$\begin{array}{l}\text { Dark } \\
\text { green }\end{array}$} & \multirow[t]{4}{*}{13.} & Pod: Shelling percentage & \multirow{4}{*}{$\begin{array}{l}\text { Medium } \\
(>75 \%)\end{array}$} \\
\hline & Light green & & & Low $(<66)$ & \\
\hline & Green & & & Medium (66-75) & \\
\hline & Dark green & & & $\operatorname{High}(>75)$ & \\
\hline \multirow{4}{*}{$\begin{array}{l}4 . \\
(+)\end{array}$} & Stem: Pubescence & \multirow{4}{*}{ Medium } & \multirow{3}{*}{$\begin{array}{ll}14 . \\
(*)\end{array}$} & Testa: Colour & \multirow[t]{3}{*}{ Uniform } \\
\hline & Absent & & & Uniform & \\
\hline & Sparse & & & Variegated & \\
\hline & Medium & & \multirow[t]{10}{*}{$\begin{array}{l}15 \\
(*)\end{array}$} & $\begin{array}{l}\text { Kernel: Colour of testa (varieties with } \\
\text { monochrome testa only) }\end{array}$ & \multirow[t]{10}{*}{ Tan (12 E 4) } \\
\hline \multirow{4}{*}{$\begin{array}{l}5 . \\
(*) \\
(+)\end{array}$} & Flower: Presence & \multirow[t]{4}{*}{ Absent } & & \begin{tabular}{|l|l|} 
& White (1 A 1$)$ \\
\end{tabular} & \\
\hline & on main axis & & & Off white(1 A 2) & \\
\hline & Absent & & & Tan (12 E 4) & \\
\hline & Present & & & Rose (Grayish red 8 B 3) & \\
\hline \multirow[t]{4}{*}{$\begin{array}{l}\text { 6. }(*) \\
(+)\end{array}$} & $\begin{array}{l}\text { Flower: Arrangement on side } \\
\text { branches }\end{array}$ & Irregular & & Purple (14 F 4) & \\
\hline & Sequential & & & Dark purple (14 F 7) & \\
\hline & Alternate & & & Salmon (6 A 4) & \\
\hline & Irregular & & & $\operatorname{Red}(10$ B 7) & \\
\hline 7. & Inflorescence & Simple & & Dark red (11 C 8) & \\
\hline$(+)$ & Simple & & 16. & Kernel: Shape & Cylindrical \\
\hline & Compound & & $(+)$ & Spheroid & \\
\hline & & & $(*)$ & Cylindrical & \\
\hline 8. $(*)$ & Time of maturity(For curing) & & & Fusiform & \\
\hline & Very early (<90 days) & & 17. $(*)$ & $\begin{array}{l}\text { Kernel: Weight of } 100 \text { kernels (about 9\% } \\
\text { moisture) }\end{array}$ & $\begin{array}{l}\text { Medium } \\
(45 \text { to } 50 \mathrm{~g})\end{array}$ \\
\hline & Early (90-100 days) & Medium & & Low $(<36 \mathrm{~g})$ & \\
\hline & Medium (101-120 days) & $(115-120$ & & Medium $(36-50 \mathrm{~g})$ & \\
\hline & Very late $(>120$ days $)$ & days) & & $\operatorname{High}(51-65 \mathrm{~g})$ & \\
\hline & & & & Very high $(>65 \mathrm{~g})$ & \\
\hline 9. & Pod: Constriction & Medium & 18. $(*)$ & Seed: Fresh seed dormancy & Present \\
\hline$(*)$ & Absent & $(5)$ & & Absent & (26 days) \\
\hline$(+)$ & Shallow & & & Present & \\
\hline & Medium & & 19. & Kernel: Oil percentage & High \\
\hline & Deep & & & Low $(<45 \%)$ & $(50.01 \%)$ \\
\hline 10. & Pod: Reticulation & Medium & & Medium (45-48\%) & \\
\hline$(*)$ & Absent & & & High (49-52\%) & \\
\hline$(+)$ & Medium & & & Very high $(>52 \%)$ & \\
\hline & Prominent & & & & \\
\hline
\end{tabular}


Table.2 Yield performance of Konkan Bhuratna in different trials conducted at different locations during 2012-13 to 2015-16

\begin{tabular}{|c|c|c|c|c|c|}
\hline \multirow[b]{2}{*}{$\begin{array}{l}\text { Sr. } \\
\text { No. }\end{array}$} & \multirow[b]{2}{*}{ Trials } & \multirow[b]{2}{*}{ Year/Season/Location } & \multicolumn{2}{|c|}{ Average pod yield (Kg/ha) } & \multirow{2}{*}{$\begin{array}{c}\text { \% Increase } \\
\text { over } \\
\text { Check }\end{array}$} \\
\hline & & & $\begin{array}{c}\text { Konkan } \\
\text { Bhuratna }\end{array}$ & Check & \\
\hline 1. & Station Trial (Rabi) & $\begin{array}{l}2012-13 \text { to } 2014-15 \\
\text { (3 seasons) }\end{array}$ & 4858 & 3577 & 35.81 \\
\hline 2. & Station Trial (Kharif) & $\begin{array}{c}2013 \text { to } 2015 \\
\text { (3 seasons) }\end{array}$ & 4358 & 2753 & 58.30 \\
\hline 3. & $\begin{array}{l}\text { University Trial } \\
\text { (Rabi) }\end{array}$ & $\begin{array}{c}2012-13 \text { to } 2013-14 \\
\text { (2 seasons \& } 7 \text { locations) }\end{array}$ & 3731 & 3114 & 19.81 \\
\hline 4. & $\begin{array}{l}\text { University Trial } \\
\text { (Kharif) }\end{array}$ & $\begin{array}{c}2013 \text { to } 2014 \\
\text { (2 seasons \& } 7 \text { locations) }\end{array}$ & 3281 & 2458 & 33.48 \\
\hline 5 & State SMVT (Kharif) & $\begin{array}{c}2013 \text { to } 2016 \\
\text { (4 seasons \& } 6 \text { locations) }\end{array}$ & 2855 & 2013 & 41.83 \\
\hline 6. & $\begin{array}{l}\text { AICRP-G Trial } \\
\text { (Kharif) }\end{array}$ & $\begin{array}{c}2016 \\
\text { (1 Season \& } 9 \text { locations) }\end{array}$ & 2349 & 1804 & 30.24 \\
\hline 7. & Adaptive Trial (Rabi) & $\begin{array}{c}2015-16 \\
\text { (5 districts \& } 21 \text { locations) }\end{array}$ & 2662 & 2216 & 20.13 \\
\hline 8. & $\begin{array}{l}\text { Adaptive Trial } \\
\text { (Kharif) }\end{array}$ & $\begin{array}{c}2015-16 \\
\text { (5 districts \& } 20 \text { locations) }\end{array}$ & 3108 & 2039 & 52.43 \\
\hline 9. & $\begin{array}{l}\text { Agronomical Trial } \\
\text { (Rabi) }\end{array}$ & $2015-16$ & 4850 & 3472 & 39.69 \\
\hline 10. & $\begin{array}{l}\text { Agronomical Trial } \\
\text { (Kharif) }\end{array}$ & 2016 & 4634 & 3393 & 36.58 \\
\hline & & verage & 3669 & 2684 & 36.69 \\
\hline
\end{tabular}

Table.3 Yield performance of Konkan Bhuratna (RTNG-29) in State Multilocation Varietal Trials (SMVT) at different locations during 2013 to 2016 in Maharashtra state

\begin{tabular}{|c|c|c|c|c|c|c|}
\hline Year & Locations & RTNG-29 & $\begin{array}{c}\text { Zonal Ch. } \\
\text { AK 159 }\end{array}$ & $\begin{array}{c}\text { Local Ch. } \\
\text { TKG Bold }\end{array}$ & $\begin{array}{c}\text { \% increased } \\
\text { over Zonal } \\
\text { check }\end{array}$ & $\begin{array}{c}\text { \% increased } \\
\text { over Local } \\
\text { check }\end{array}$ \\
\hline 2013 & 6 & 2935 & 2240 & 2039 & 31.02 & 43.94 \\
\hline 2014 & 7 & 2349 & 1907 & 2048 & 23.17 & 14.70 \\
\hline 2015 & 5 & 3061 & 2281 & 1762 & 34.20 & 73.72 \\
\hline 2016 & 7 & 3075 & 2347 & 2203 & 31.02 & 39.58 \\
\hline \multicolumn{2}{|c|}{ Pooled } & $\mathbf{2 8 5 5}$ & $\mathbf{2 1 9 4}$ & $\mathbf{2 0 1 3}$ & $\mathbf{3 0 . 1 4}$ & $\mathbf{4 1 . 8 3}$ \\
\hline
\end{tabular}

Table.4 Yield performance of RTNG-29 in AICRP-G Trials at at various location in Zone $\mathrm{V}^{\text {th }}$ during Kharif 2016

\begin{tabular}{|c|l|c|c|c|c|}
\hline S.N. & \multicolumn{1}{|c|}{ Entry } & Mean & $\begin{array}{c}\text { \% increased } \\
\text { over ICGS } \\
\mathbf{7 6}(\mathbf{Z C})\end{array}$ & $\begin{array}{c}\text { \% increased } \\
\text { over } \\
\text { GG 16 (ZC) }\end{array}$ & $\begin{array}{c}\text { \% increased } \\
\text { over ICGV } \\
\text { 00348 (ZC) }\end{array}$ \\
\hline $\mathbf{1}$ & Konkan Bhuratna (RTNG 29) & 2349 & 30.24 & 45.68 & 34.64 \\
\hline $\mathbf{2}$ & ICGS 76 (ZC) & 1804 & & & \\
\hline $\mathbf{3}$ & GG 16 (ZC) & 1613 & & & \\
\hline $\mathbf{4}$ & ICGV 00348 (ZC) & 1745 & & & \\
\hline
\end{tabular}


Table.5 Performance of Konkan Bhuratna under adaptive trials conducted on farmer's field during Rabi 2015-16 and Kharif 2016

\begin{tabular}{|c|c|c|c|c|c|}
\hline Year & $\begin{array}{c}\text { No. of } \\
\text { Trials }\end{array}$ & $\begin{array}{c}\text { No. of } \\
\text { District }\end{array}$ & RTNG-29 & $\begin{array}{c}\text { Check } \\
\text { TKG-Bold }\end{array}$ & $\begin{array}{c}\text { \% Increase } \\
\text { over check }\end{array}$ \\
\hline Rabi $2015-16$ & 21 & 05 & 2662 & 2216 & $\mathbf{2 0 . 1 3}$ \\
\hline Kharif 2016 & 20 & 05 & 3108 & 2039 & $\mathbf{5 2 . 4 3}$ \\
\hline
\end{tabular}

Table.6 Pod yield of groundnut as influenced by genotypes/varieties, spacing and different fertilizer levels Rabi 2015-16

\begin{tabular}{|c|c|c|c|c|c|}
\hline \multirow[b]{2}{*}{ Genotypes } & \multirow[b]{2}{*}{ Spacing } & \multicolumn{4}{|c|}{ Fertilizer levels } \\
\hline & & $\begin{array}{c}\mathbf{F}_{\mathbf{0}} \\
(00: 00: 00 \mathrm{~kg} \mathrm{NPK} / \mathrm{ha})\end{array}$ & $\begin{array}{c}\mathbf{F}_{\mathbf{1}} \\
(25: 50: 00 \mathrm{~kg} \mathrm{NPK} / \mathrm{ha})\end{array}$ & $\begin{array}{c}\mathbf{F}_{\mathbf{2}} \\
(30: 70: 00 \mathrm{~kg} \mathrm{NPK} / \mathrm{ha})\end{array}$ & Mean \\
\hline \multirow{3}{*}{$\begin{array}{c}\mathrm{V}_{1} \\
\text { (RTNG- } \\
\text { 29) }\end{array}$} & $\mathbf{S}_{\mathbf{1}}(30 \times 10 \mathrm{~cm})$ & 23.07 & 39.79 & 48.50 & 37.12 \\
\hline & $\mathbf{S}_{2}(30 \times 15 \mathrm{~cm})$ & 22.41 & 33.58 & 37.93 & 31.30 \\
\hline & $\mathbf{S}_{\mathbf{3}}(45 \times 10 \mathrm{~cm})$ & 23.58 & 30.04 & 33.64 & 29.09 \\
\hline \multirow{4}{*}{$\begin{array}{c}\mathrm{V}_{2} \\
\text { (TKG- } \\
\text { Bold) }\end{array}$} & $\mathbf{S}_{\mathbf{1}}(30 \times 10 \mathrm{~cm})$ & 20.49 & 29.33 & 34.72 & 28.18 \\
\hline & $\mathbf{S}_{\mathbf{2}}(30 \times 15 \mathrm{~cm})$ & 19.41 & 22.17 & 30.94 & 24.17 \\
\hline & $\mathbf{S}_{\mathbf{3}}(45 \times 10 \mathrm{~cm})$ & 17.46 & 25.74 & 31.61 & 24.93 \\
\hline & Mean & 21.07 & 30.11 & 36.22 & 29.13 \\
\hline
\end{tabular}

Table.7 Pod yield of groundnut as influenced by genotypes/varieties, spacing and different fertilizer levels Kharif- 2016

\begin{tabular}{|c|c|c|c|c|c|}
\hline \multirow[b]{2}{*}{ Genotypes } & \multirow[b]{2}{*}{ Spacing } & \multicolumn{4}{|c|}{ Fertilizer levels } \\
\hline & & $\begin{array}{c}\mathbf{F}_{\mathbf{0}} \\
(00: 00: 00 \mathrm{~kg} \mathrm{NPK} / \mathrm{ha})\end{array}$ & $\begin{array}{c}\mathbf{F}_{\mathbf{1}} \\
(25: 50: 00 \mathrm{~kg} \mathrm{NPK} / \mathrm{ha})\end{array}$ & $\begin{array}{c}\mathbf{F}_{\mathbf{2}} \\
(30: 70: 00 \mathrm{~kg} \text { NPK/ha })\end{array}$ & Mean \\
\hline \multirow{3}{*}{$\begin{array}{c}\mathrm{V}_{1} \\
\text { (RTNG- } \\
29) \\
\end{array}$} & $\mathbf{S}_{\mathbf{1}}(30 \times 10 \mathrm{~cm})$ & 25.48 & 37.96 & 46.34 & 36.59 \\
\hline & $\mathbf{S}_{\mathbf{2}}(30 \times 15 \mathrm{~cm})$ & 22.68 & 27.14 & 31.54 & 27.12 \\
\hline & $\mathbf{S}_{\mathbf{3}}(45 \times 10 \mathrm{~cm})$ & 23.77 & 26.50 & 29.82 & 26.70 \\
\hline \multirow{4}{*}{$\begin{array}{c}\mathrm{V}_{2} \\
\text { (TKG- } \\
\text { Bold) }\end{array}$} & $\mathbf{S}_{\mathbf{1}}(30 \times 10 \mathrm{~cm})$ & 23.13 & 29.68 & 33.93 & 28.91 \\
\hline & $\mathbf{S}_{\mathbf{2}}(30 \times 15 \mathrm{~cm})$ & 21.09 & 23.75 & 29.06 & 24.63 \\
\hline & $\mathbf{S}_{\mathbf{3}}(45 \times 10 \mathrm{~cm})$ & 21.49 & 22.89 & 26.72 & 23.70 \\
\hline & Mean & 22.94 & 27.99 & 32.90 & 27.94 \\
\hline
\end{tabular}

Table.8 Quality analysis of Konkan Bhuratna (RTNG-29) culture (Test Report of Gujarat Laboratory)

\begin{tabular}{|l|c|c|c|c|c|c|c|c|}
\hline \multirow{2}{*}{$\begin{array}{l}\text { Name of the } \\
\text { culture / check }\end{array}$} & $\begin{array}{c}\text { Oil } \\
(\%)\end{array}$ & $\begin{array}{c}\text { Protein } \\
(\%)\end{array}$ & $\begin{array}{c}\text { Palmitic } \\
\text { Acid } \\
(\%)\end{array}$ & $\begin{array}{c}\text { Steric } \\
\text { Acid } \%)\end{array}$ & $\begin{array}{c}\text { Oleic } \\
\text { Acid } \\
(\%)\end{array}$ & $\begin{array}{c}\text { Linoleic } \\
\text { Acid } \\
(\%)\end{array}$ & $\begin{array}{c}\text { Arachidic } \\
\text { Acid }(\%)\end{array}$ & $\begin{array}{c}\text { Bahenic } \\
\text { Acid } \\
(\%)\end{array}$ \\
\hline RTNG-29 & 50.01 & 22.44 & 14.60 & 3.20 & 46.08 & 31.71 & 0.78 & 2.28 \\
\hline TKG-Bold & 48.11 & 24.56 & 13.09 & 2.82 & 45.81 & 33.16 & 1.12 & 2.76 \\
\hline Konkan Gaurav & 47.12 & 24.05 & 13.96 & 3.23 & 49.28 & 29.25 & 0.83 & 2.18 \\
\hline
\end{tabular}


Table.9 Reaction to major diseases on the basis of pooled mean (Rb-2012-13 to Rb-2014-15)

\begin{tabular}{|c|c|c|c|c|c|c|c|c|c|c|}
\hline Entry & \multicolumn{2}{|c|}{$\begin{array}{c}\text { ELS } \\
(0-9) \\
\text { Scale }\end{array}$} & \multicolumn{2}{|c|}{$\begin{array}{c}\text { LLS } \\
\text { (0-9) Scale }\end{array}$} & \multicolumn{2}{|c|}{$\begin{array}{c}\text { Rust } \\
\text { (0-9) Scale }\end{array}$} & \multicolumn{2}{|c|}{$\begin{array}{l}\text { PBND } \\
(\%)\end{array}$} & \multicolumn{2}{|c|}{$\begin{array}{c}\text { Alterneria leaf } \\
\text { blight (0-9) Scale }\end{array}$} \\
\hline Date of appearance & \multicolumn{2}{|c|}{$\begin{array}{l}20-25 \\
\text { Days }\end{array}$} & \multicolumn{2}{|c|}{ 55-60 Days } & \multicolumn{2}{|c|}{ 62-65 Days } & \multicolumn{2}{|c|}{ 40-45 Days } & \multicolumn{2}{|c|}{ 45-60 Days } \\
\hline RTNG-29 & 0.0 & $\mathrm{HR}$ & 1.0 & $\mathrm{R}$ & 1.3 & MR & 1.5 & $\mathrm{R}$ & 1 . & $\mathrm{R}$ \\
\hline TKG Bold (Ch-I) & 0.0 & $\mathrm{HR}$ & 3.2 & MS & 1.6 & MR & 2.8 & MR & 2. & MR \\
\hline Konkan Gaurav (Ch-II) & 0.0 & HR & 2.6 & $\mathrm{MS}$ & 2.8 & MS & 2.6 & MR & 2.7 & MR \\
\hline
\end{tabular}

Table.10 Reaction to major diseases on the basis of pooled mean (Kh-2013 to Kh-2015)

\begin{tabular}{|c|c|c|c|c|c|c|c|c|c|c|}
\hline Entry & \multicolumn{2}{|c|}{$\begin{array}{c}\text { ELS } \\
\text { (0-9) Scale }\end{array}$} & \multicolumn{2}{|c|}{$\begin{array}{c}\text { LLS } \\
(0-9) \text { Scale }\end{array}$} & \multicolumn{2}{|c|}{$\begin{array}{c}\text { Rust } \\
\text { (0-9) Scale }\end{array}$} & \multicolumn{2}{|c|}{$\begin{array}{c}\text { PBND } \\
(\%)\end{array}$} & \multicolumn{2}{|c|}{$\begin{array}{c}\text { Alterneria leaf } \\
\text { blight (0-9) } \\
\text { Scale }\end{array}$} \\
\hline Date of ap! & $20-2$ & Days & $55-6$ & Days & $62-6$ & Days & $40-4$ & Days & & Days \\
\hline RTNG-29 & 0.0 & $\mathrm{HR}$ & 1.0 & $\mathrm{R}$ & 1.2 & MR & 1.6 & $\mathrm{R}$ & 1.4 & $\mathrm{R}$ \\
\hline TKG Bold (Cl & 0.0 & HR & 2.3 & MR & 2.0 & MR & 2.7 & MR & 2.2 & MR \\
\hline Konkan Gaurav (Ch-II) & 0.0 & HR & 3.0 & MS & 3.2 & MS & 2.7 & MR & 2.4 & MR \\
\hline
\end{tabular}

Table.11 Reaction to major Insect pests on the basis of pooled mean

( $\mathrm{Rb}-2012-13$ to $\mathrm{Rb}-2014-15)$

\begin{tabular}{|c|c|c|c|c|c|c|c|c|c|c|}
\hline \multirow{2}{*}{ Entry } & \multicolumn{3}{|c|}{ Thrips } & \multicolumn{3}{c|}{ Jassids } & \multicolumn{2}{c|}{ Leaf miner } & \multicolumn{2}{c|}{ Defoliator } \\
\cline { 2 - 11 } & 30D & 60D & 90D & 30D & 60D & 90D & 30D & 60D & 90D & 30D \\
\hline RTNG-29 & 1.6 & 1.9 & 0.9 & 1.1 & 1.0 & 0.9 & 1.1 & 1.6 & 1.0 & 1.5 \\
\hline Reaction & HR & HR & HR & HR & HR & HR & HR & HR & HR & HR \\
\hline TKG-Bold (Ch-I) & 4.6 & 5.2 & 4.6 & 2.8 & 2.2 & 1.5 & 4.4 & 5.4 & 4.9 & 6.6 \\
\hline Reaction & MS & S & MS & R & R & HR & MR & MS & MS & S \\
\hline Konkan Gaurav (Ch-II) & 3.0 & 3.2 & 3.8 & 2.2 & 1.9 & 1.7 & 3.2 & 4.5 & 4.0 & 3.2 \\
\hline Reaction & MR & MR & MR & R & HR & HR & MR & MR & MS & MR \\
\hline
\end{tabular}

Table.12 Reaction to major Insect pests on the basis of pooled mean (Kh-2013 to Kh-2015)

\begin{tabular}{|r|c|c|c|c|c|c|c|c|c|c|}
\hline \multirow{2}{*}{ Entry } & \multicolumn{3}{|c|}{ Thrips } & \multicolumn{3}{c|}{ Jassids } & \multicolumn{3}{c|}{ Leaf miner } & \multicolumn{2}{c|}{ Defoliator } \\
\cline { 2 - 11 } \multicolumn{1}{|c|}{ RTNG-29 } & 30D & 60D & 90D & 30D & 60D & 90D & 30D & 60D & 90D & 30D \\
\hline Reaction & R & HR & R & HR & HR & HR & HR & HR & HR & HR \\
\hline TKG-Bold (Ch-I) & 5.3 & 4.7 & 4.4 & 3.3 & 2.6 & 2.2 & 3.2 & 4.7 & 4.7 & 5.1 \\
\hline Reaction & S & MS & MS & R & R & R & MR & MR & MS & S \\
\hline Konkan Gaurav (Ch-II) & 3.5 & 3.3 & 3.6 & 2.6 & 2.0 & 2.6 & 3.6 & 3.3 & 3.7 & 3.3 \\
\hline Reaction & MR & MR & MR & R & R & R & MR & MR & MR & MR \\
\hline
\end{tabular}


In state multilocation varietal trials conducted during Kharif 2013, Kharif 2014, Kharif 2015 and Kharif 2016 in these 4 seasons, and six different locations, Konkan Bhuratna (RTNG29) has recorded $31.02 \%, 23.17 \%, 34.20 \%$ and $31.02 \%$ increased pod yield over zonal check AK-159 respectively, while as it was recorded $43.94 \%, 14.70 \%, 73.72 \%$ and $39.58 \%$ higher pod yield over local check TKG-Bold, respectively. On the basis of pooled mean performance of state multilocation trial, it showed $30.14 \%$ and $41.83 \%$ increased pod yield over zonal and local check respectively, Table 3 (RRC Report 2014, RRC Report 2015 and RRC Report 2016).

Konkan Bhurtna was evaluated in All India Coordinated Trials testing code at nine different locations during Kharif- 2016 in the country. It showed $30.24 \%, 45.68 \%$ and $34.64 \%$ increase in pod yield over Zonal Checks, ICGS 76, GG 16 and ICGV 00348 respectively conducted during Kharif-2016 season indicating it's wider adaptability in varied agro-ecological situations in the country (Table 4) (DGR Report, 2017).

The groundnut culture, Konkan Bhuratna (2662 and $3108 \mathrm{~kg} / \mathrm{ha}$ ) has recorded $20.13 \%$ and $52.43 \%$ increase in pod yield over check TKG-Bold (2216 and $2039 \mathrm{~kg} / \mathrm{ha}$ ) in 21 and 20 adaptive trials conducted in five district during Rabi 2015-16 and Kharif 2016 respectively (Table 5).

The Agronomical experiment was conducted at ARS, Shirgaon (Ratnagiri) to study the effect of spacing and different nitrogen levels along on promising groundnut entry, Konkan Bhuratna during Rabi 2015-16, recorded higher pod yield $4850 \mathrm{~kg} / \mathrm{ha}$ by adopting $30 \mathrm{x}$ $10 \mathrm{~cm}$ spacing and 30:70:00 NPK $\mathrm{kg} / \mathrm{ha}$ as fertilizer dose, which was $39.69 \%$ higher over cultivar TKG-Bold (3472 kg/ha) by adopting same package of practice (Table 6).
In Agronomical trial, conducted during Rabi season, promising groundnut entry, Konkan Bhuratna given higher pod yield $4634 \mathrm{~kg} / \mathrm{ha}$ by adopting $30 \mathrm{x} 10 \mathrm{~cm}$ spacing and 30:70:00 $\mathrm{NPK} \mathrm{kg} / \mathrm{ha}$ as fertilizer dose, which was $36.58 \%$ higher over cultivar TKG-Bold (3393 $\mathrm{kg} / \mathrm{ha}$ ) by adopting same package of practice (Table 7).

The oil quantity and other quality analysis of Konkan Bhuratna groundnut variety was estimated at the Gujrat Laboratory during the year-2016.

It showed high oil content $(50.01 \%)$, protein content (23.44\%), Palmitic Acid (14.60\%), Steric Acid (3.20\%), Oleic Acid (46.08\%), Linoleic Acid (31.71\%), Arachidic Acid (0.78 $\%)$ Bahenic Acid (2.28\%) (Table 8).

Konkan Bhuratna is resistant to early and late leaf spot, rust, PBND, alterneria leaf blight diseases and also resistant to thrips, jassids, leaf miner, defoliator insect and pests (Table 9-12).

Konkan Bhurtana, groundnut variety is dwarf in height $(25-30 \mathrm{~cm})$, medium in duration (115-120 days), having good shelling percentage (kharif 73.5-76.0\% and rabi 70.2$76.3 \%$ ), medium bold type (100 kernel weight (45-50 g), having fresh seed dormancy (26 days), high oil content (kharif 50.02-50.08\% and rabi 49.70-50.14\%), resistant to thrips, jassids, leaf miner and defoliator insect pests and resistant to early, late leaf spot, rust, PBND and alterneria leaf blight diseases. It is suitable for groundnut growing areas of Maharashtra.

Therefore the groundnut variety Konkan Bhuratna recommended for release as a commercially cultivation in upland area during kharif and irrigated area during rabi season in Konkan region of the Maharashtra state in the year 2017. 


\section{Acknowledgement}

The authors are grateful to the Directorate of Groundnut Research, Junaghadh, Gujarat for providing the research material and financial support for development of this variety through All India Coordinated Research Project on groundnut (AICRP-G) programme. They are also thankful to the scientists and the technical staff who helped in screening and evaluation of above variety at in different locations.

\section{References}

Anonymous, 2017. Release proposal of Konkan Bhuratna, presented during Joint Agresco, 2017 held on 28-31 $1^{\text {st }}$ May, 2017 at VNMAU, Parbhani.
Arya, S.S., Salve, A.R. and Chauhan, S. 2016. Peanut as functional food: a review $J$. Food Sci Technol., 53: 31-41.

DGR Report, 2016. Annual groundnut Workshop report, 25-27 April-2017.

FAO, 2015. www.faostate.com

Panse, V.G. and Sukhatme P.V., 1967. Statistical Methods for Agricultural Workers. ICAR. Publ. New Delhi (India).

RRC Report, 2014. Research Review Meeting Field Crops-Oilseeds 10-11 ${ }^{\text {th }}$ March 2014, Dr. BSKKV, Dapoli, (MS).

RRC Report, 2015. Research Review Meeting Field Crops-Oilseeds $26-27^{\text {th }}$ March 2015, Dr. BSKKV, Dapoli, (MS).

RRC Report, 2016. Research Review Meeting Field Crops-Oilseeds 13 ${ }^{\text {th }}$ April 2016, Dr. BSKKV, Dapoli, (MS).

\section{How to cite this article:}

Waghmode, B.D., V.C. Navhale, A.S. Kamble, N.G. Sonone and Thaware, B.L. 2017. Development of Medium Duration, High Oil Content and Yield Potential Groundnut Variety Konkan Bhuratna for Konkan Region of Maharashtra State. Int.J.Curr.Microbiol.App.Sci. 6(12): 999-1006. doi: https://doi.org/10.20546/ijcmas.2017.612.111 